\title{
A New Kind of Science: Ten Years Later
}

\author{
David H. Bailey*
}

May 17, 2012

It has been ten years since Stephen Wolfram published his magnum opus $A$ New Kind of Science [14]. It is worth re-examining the book and its impact in the field.

\section{Highlights of ANKS}

The present author personally read the book with great interest when it was first published. Of particular interest then and now are the many illustrations, particularly those of the complex patterns generated by certain cellular automata systems, such as rule 30 and rule 110, as contrasted with the very regular patterns produced by other rules. In this regard, graphical analyses of these cellular automata rules join a select group of modern mathematical phenomena (including, for example, studies of the Mandlebrot set, chaotic iterations and certain topological manifolds) that have been studied graphically as well as analytically.

In looking again at ANKS, the present author is struck today, as in 2002, with the many interesting items in the endnote section, which occupies 350 pages of two-column, small-font text. In some respects, the endnotes of ANKS constitute an encyclopedia of sorts, covering, in concise yet highly readable form, historical background, mathematical foundations and scientific connections of a wide variety of topics related to modern-day computing. Much of this material remains as cogent and interesting today as when it was written over ten years ago. Some of the particularly interesting endnote passages are the following:

1. Wolfram's entry on "History of experimental mathematics" (pg. 899) contains a number of interesting insights on the practice of using computers as exploratory tools in mathematics.

2. In his entry "Randomness in markets" (pg. 1014), Wolfram notes that in modern-day financial markets, large price fluctuations are significantly more common than a Gaussian distribution would imply - a phenomenon amply affirmed in the 2007-2009 worldwide financial crash.

${ }^{*}$ Lawrence Berkeley National Laboratory, Berkeley, CA 94720, DHBailey@lbl.gov. Supported in part by the Director, Office of Computational and Technology Research, Division of Mathematical, Information, and Computational Sciences of the U.S. Department of Energy, under contract number DE-AC02-05CH11231. 
3. Under "Einstein equations" (pg. 1052-1054), Wolfram presents a brief and yet informative introduction to the equations of general relativity, together with some interesting computational perspectives in the general arena of relativity and cosmology.

4. Under "Quantum phenomena" (pg. 1056-1065), Wolfram presents an even more detailed overview of quantum mechanics, including an introduction to Feynman diagrams, quantum field theory and Bell's inequality, all with interesting connections to computation.

5. Under "Data Compression" (pg. 1069-1074), Wolfram includes numerous details of state-of-the-art data compression algorithms.

6. Beginning with a series of articles under the heading "Undecidability and Intractibility" (pg. 1136-1149), Wolfram presents a fairly technical but nonetheless quite coherent introduction to many of the topics of modern theoretical computer science, including undecidability, computational complexity, Turing machines, NP-completeness and quantum computers.

7. Immediately following the material on theoretical computer science is a similarly detailed introduction (pg. 1149-1177) to modern developments in the foundations of mathematics, with implications for computing.

8. In a fascinating section "Intelligence in the Universe" (pg. 1177-1191), Wolfram discusses such topics as the origin of life, extraterrestrial life, the nature of animal and human intelligence, Fermi's paradox and speculations, from a computational point of view, as to why our search for extraterrestrial intelligence so far as been unsuccessful.

\section{Experimental mathematics}

With regards to item 1 in the list above (experimental mathematics), it is worth pointing out that while Wolfram is very well versed in rigorous mathematical proof, nonetheless he confesses (pg. 899), "by now I have come to trust the correctness of conclusions based on simple systematic computer experiments much more than I trust all but the simplest proofs." Wolfram laments the fact that so few others in the field of modern-day mathematics are willing embrace this computational-experimental paradigm (pg. 899):

[E]ven now, unlike essentially all other branches of science, mainstream mathematics continues to be entirely dominated by theoretical rather than experimental methods. And even when experiments are done, their purpose is essentially always just to provide another way to look at traditional questions in traditional mathematical systems.

Fortunately, this is one arena where substantial progress has been made in the past ten or fifteen years. Nowadays many research mathematicians use the 
computer to manipulate symbolic expressions, generate sequences, visually inspect numerical data, check analytical work, compute expressions to very high numeric precision, and otherwise explore the mathematical universe using the latest computer technology. This trend has been greatly facilitated by continuing improvements in Mathematica and other mathematical software packages. Just as important for this phenomenon is the entry into the field of a large number of junior-level mathematicians who are completely comfortable with computer-based tools, and who instinctively look to the computer as the first step in investigating a mathematical question.

\section{A new formula for pi}

Perhaps one of the most interesting of the recent computer-discovered mathematical facts is what is now known as the "BBP" formula for $\pi=3.14159 \ldots$.

$$
\pi=\sum_{k=0}^{\infty} \frac{1}{16^{k}}\left(\frac{4}{8 k+1}-\frac{2}{8 k+4}-\frac{1}{8 k+5}-\frac{1}{8 k+6}\right),
$$

which can also be written

$$
\begin{aligned}
\pi= & 4 \sum_{k=0}^{\infty} \frac{1}{16^{k}(8 k+1)}-2 \sum_{k=0}^{\infty} \frac{1}{16^{k}(8 k+4)}-\sum_{k=0}^{\infty} \frac{1}{16^{k}(8 k+5)} \\
& -\sum_{k=0}^{\infty} \frac{1}{16^{k}(8 k+6)} .
\end{aligned}
$$

Here $\sum$ is the usual mathematical notation for summation. Thus formula (2), for instance, is merely shorthand for

$$
\begin{aligned}
\pi= & 4\left(1+\frac{1}{16(8+1)}+\frac{1}{16^{2}(2 \cdot 8+1)}+\frac{1}{16^{3}(3 \cdot 8+1)}+\cdots\right) \\
& -2\left(\frac{1}{4}+\frac{1}{16(8+4)}+\frac{1}{16^{2}(2 \cdot 8+4)}+\frac{1}{16^{3}(3 \cdot 8+4)}+\cdots\right) \\
& -\left(\frac{1}{5}+\frac{1}{16(8+5)}+\frac{1}{16^{2}(2 \cdot 8+5)}+\frac{1}{16^{3}(3 \cdot 8+5)}+\cdots\right) \\
& -\left(\frac{1}{6}+\frac{1}{16(8+6)}+\frac{1}{16^{2}(2 \cdot 8+6)}+\frac{1}{16^{3}(3 \cdot 8+6)}+\cdots\right) .
\end{aligned}
$$

These infinite series converge quite rapidly — if one adds up just the first four terms displayed above for each series (i.e., truncating the sums at the $\cdots$ sign), the result will be a value of $\pi$ correct to six digits.

However, the most remarkable feature of BBP formula is that it permits one to calculate a string of binary (base 2) or hexadecimal (base-16) digits of $\pi$ beginning at an arbitrary position $n$, without needing to calculate any of the preceding $n-1$ digits. See [4], [9] or [10, pg. 118-125] for details. Indeed, a 
Mathematica implementation of this surprisingly simple scheme is presented in ANKS (pg. 912).

Recently Tsz-Wo Sze of Yahoo! Cloud Computing demonstrated a closely related variant of this scheme by calculating binary digits of $\pi$ beginning at position two quadrillion [7]. The first 25 binary digits beginning at this point are: 0111001101100000100101001.

An even more interesting aspect of the BBP formula, one particularly relevant to the present discussion, is the fact that it was discovered by a computer. Indeed, it may be the first instance in the history of mathematics where a significant new formula for $\pi$ was found by a computer.

This all happened in 1995, when Canadian mathematician Peter Borwein was considering whether or not it was possible to calculate the $n$-th digit of a mathematical constant such as $\pi$ by some shortcut that avoided the necessity of computing all digits up to and including the $n$-th digit. He and Simon Plouffe found a way to compute the $n$-th binary digit of the natural logarithm of two, namely $\ln 2=0.693147 \ldots$, by manipulating the following well-known formula for $\ln 2$ :

$$
\ln 2=\sum_{k=1}^{\infty} \frac{1}{k 2^{k}}=\frac{1}{2}+\frac{1}{3 \cdot 2^{3}}+\frac{1}{4 \cdot 2^{4}}+\frac{1}{5 \cdot 2^{5}}+\cdots
$$

After this discovery, Borwein and Plouffe immediately asked whether they could do the same mathematical "trick" for $\pi$. It all depended on finding a similar formula for $\pi$. Peter Borwein, who was very familiar with the mathematical literature regarding $\pi$, was not aware of any such formula for $\pi$, and it seemed exceedingly unlikely that such a formula would have escaped detection by the many thousands of great mathematicians who have studied $\pi$ through the ages. But Plouffe embarked on a computer search for such a formula, using a 200-digit computer implementation (provided by the present author) of mathematiciansculptor Helaman Ferguson's integer relation "PSLQ" algorithm, which finds integer linear relations among an input set of numerical values. After several months of fits and starts, Plouffe and his computer found formula (1). The rest, as they say, is history.

Since 1995, researchers have discovered similar digit-calculating formulas for numerous other fundamental constants of mathematics, in most cases by similar computer searches using the PSLQ algorithm. See [10, Chap. 3] or [7] for details.

\section{Ramanujan's continued fraction}

Srinivasa Ramanujan (1887-1920), born to a poor family in India, learned mathematics mostly by studying math books on his own. His genius was recognized by British mathematician G. H. Hardy, who invited him to come work with him in Cambridge. Ramanujan's mathematical achievements have been recognized as among the greatest of all time, in spite of the fact that he died at the tender age of 32 . 
One of the many topics that he addressed in his notebooks is the following class of continued fractions. Given $a, b, \eta>0$, define

$$
R_{\eta}(a, b)=\frac{a}{\eta+\frac{b^{2}}{\eta+\frac{4 a^{2}}{\eta+\frac{9 b^{2}}{\eta+} \cdot}}} .
$$

This complicated-looking expression simply means to evaluate the indicated compound fraction out to some level, and then take the limit as more and more terms are included. Ramanujan discovered the beautiful fact that

$$
\frac{R_{\eta}(a, b)+R_{\eta}(b, a)}{2}=R_{\eta}\left(\frac{a+b}{2}, \sqrt{a b}\right),
$$

for certain $a, b>0$ parameterized by elliptic functions. And indeed this is true for all $a, b>0$, as Berndt notes in his annotation of Ramanujan's notebook [8].

Just as ANKS was being completed, a group of mathematicians (including Jonathan Borwein, Richard Crandall, David Borwein, Raymond Mayer and others) applied the tools of experimental mathematics to study these continued fractions. This started with a simple attempt to numerically validate (6), which, in turn, meant numerically computing formula (5).

Unfortunately, a first attempt to numerically compute $R_{1}(1,1)$, as a prototype problem, failed miserably - after a lengthy computation only three reliable digits were produced: $0.693 \ldots$. But researchers recognized this value as close to the value of $\ln 2=0.693147 \ldots$, and then discovered that convergence of the continued fraction is worst when $a=b$ (i.e., the initial problem they selected was a poor choice).

Eventually a number of very interesting results were obtained, including an algorithm to compute (5) in the complex plane, and so to determine exactly when it converged. For instance, with the help of Maple and Mathematica and a scatter plot, these researchers discovered that the fraction converges and (6) holds exactly when $(a, b)$ lie in the cardioid defined by $|a|+|b| \geq 2 \sqrt{|a||b|}$. They then determined an elliptic function representation from which the simple formula

$$
\mathcal{R}_{1}(a, a)=2 \int_{0}^{1} \frac{t^{1 / a}}{1+t^{2}} d t,
$$

true for all nonzero real numbers $a$, followed easily. No such formula is known for $\mathcal{R}_{\eta}(a, b)$ with $a \neq b$ or not real, although striking results have been obtained in cases such as $\mathcal{R}_{\eta}(i a, i a)$, for real $a$, which exhibits true chaos [2].

Study of convergence of these Ramanujan continued fractions was facilitated by reducing them to the following discrete dynamical system: Given complex numbers $a$ and $b$ as in (5), set $t_{0}=1, t_{1}=1$ and then iterate

$$
t_{n}:=\frac{1}{n}+\omega_{n-1}\left(1-\frac{1}{n}\right) t_{n-2},
$$


where $\omega_{n}=a^{2}$ or $b^{2}$, depending on whether $n$ is even or odd. It can be shown that $\mathcal{R}_{\eta}(a, b)$ diverges if and only if the sequence $\sqrt{n} t_{n}$ remains bounded.

If one studies this iteration based solely on its numerical values, nothing much is evident - one only sees that $t_{n} \rightarrow 0$ fairly slowly. However, if one looks at this iteration pictorially, significantly more can be learned. In particular, if one plots these iterates in the complex plane, scaled by $\sqrt{n}$, with iterations colored blue or red depending on odd or even $n$, then some remarkable fine structure appears - see Figure 1.

With assistance of such plots, the behavior of these iterates (and the Ramanujan continued fractions themselves) is now quite well understood. A Cinderella applet exploring the dynamics of these iterations is available at http: //carma.newcastle.edu.au/jon/dynamics.html. When $a$ and $b$ are complex numbers such that $|a|=|b|=1$, a circle appears when either of $a, b$ is not a root of unity, but $k$ isolated spirals are seen when one of them is a $k$-th root of unity (i.e., when $a=e^{2 i \pi / k}$ for some integer $k$ ).

In short, Ramanujan continued fractions and related iterations join the distinguished category of mathematical objects that have been profitably studied via computer graphics, in company with Wolfram's cellular automata, chaotic sequences, and the Julia-set structures of roots of algebraic equations.

\section{Formulas for the Riemann zeta function}

The Riemann zeta function, which is defined by the simple formula

$$
\zeta(s)=\sum_{k=0}^{\infty} \frac{1}{n^{s}}=1+\frac{1}{2^{s}}+\frac{1}{3^{s}}+\frac{1}{4^{s}}+\cdots,
$$

is one of the most important objects in modern mathematics, with applications in physics, probability theory, applied statistics and number theory. A premier unsolved problem of mathematics, for which the Clay Mathematics Institute has offered US $\$ 1,000,000$ for solution, is to rigorously prove the "Riemann hypothesis," namely the assertion that all of the nontrivial solutions of the equation $\zeta(s)=0$ in the complex plane lie precisely on a particular vertical straight line.

Among the many questions explored by mathematicians over the past century regarding the Riemann zeta function are whether the following intriguing formulas, which have been known for several decades, can be generalized:

$$
\begin{aligned}
& \zeta(2)=3 \sum_{k=1}^{\infty} \frac{1}{k^{2}\left(\begin{array}{c}
2 k \\
k
\end{array}\right)}=3\left(\frac{1}{2}+\frac{1}{4 \cdot 6}+\frac{1}{9 \cdot 20}+\cdots\right) \\
& \zeta(3)=\frac{5}{2} \sum_{k=1}^{\infty} \frac{(-1)^{k+1}}{k^{3}\left(\begin{array}{c}
2 k \\
k
\end{array}\right)}=\frac{5}{2}\left(-\frac{1}{1 \cdot 2}+\frac{1}{8 \cdot 6}-\frac{1}{27 \cdot 20}+\cdots\right) \\
& \zeta(4)=\frac{36}{17} \sum_{k=1}^{\infty} \frac{1}{k^{4}\left(\begin{array}{c}
2 k \\
k
\end{array}\right)}=\frac{36}{17}\left(\frac{1}{1 \cdot 2}+\frac{1}{16 \cdot 6}+\frac{1}{81 \cdot 20}+\cdots\right) .
\end{aligned}
$$




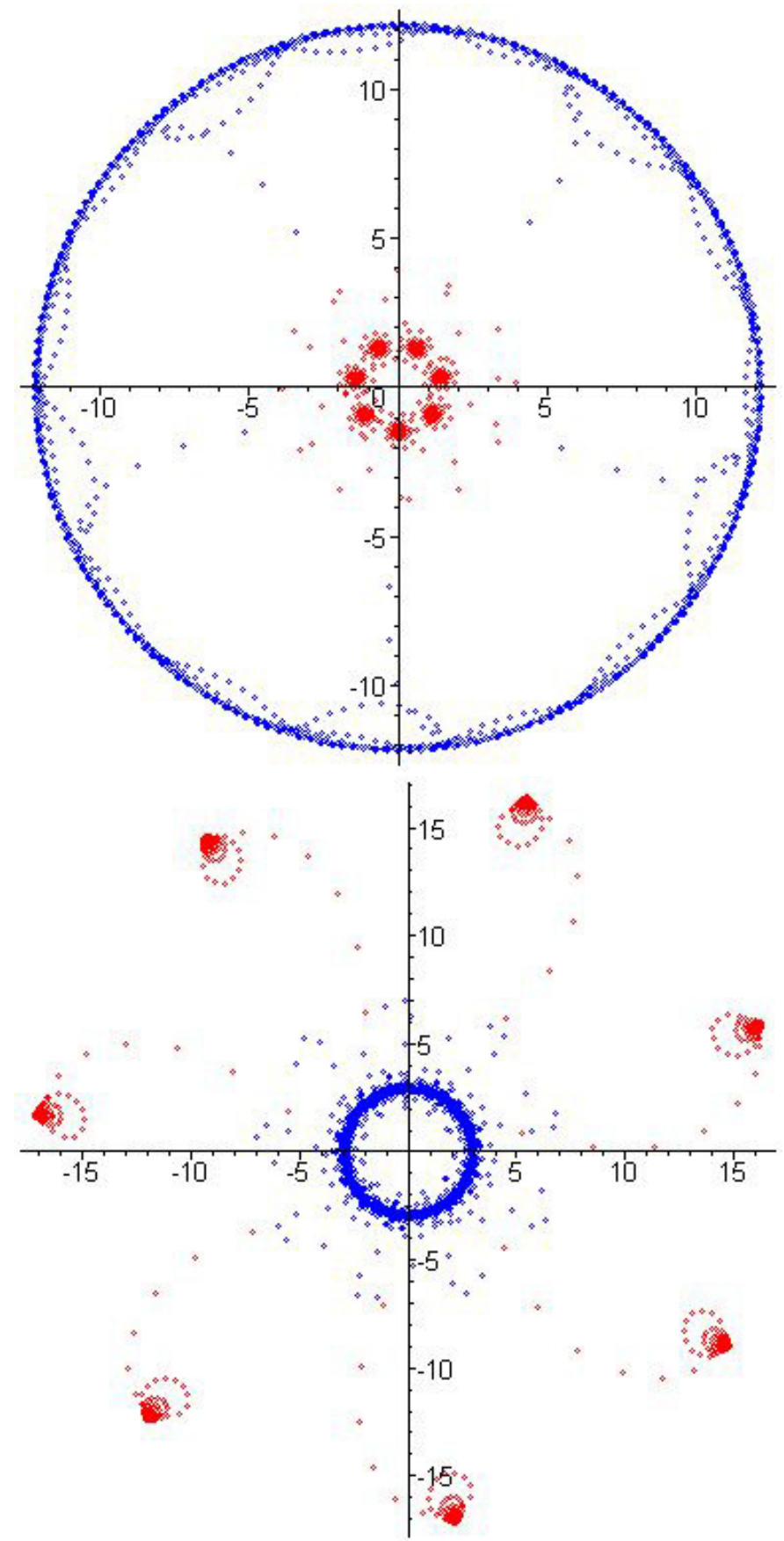

Figure 1: Dynamics and attractors discovered by plotting $\sqrt{n} t_{n}$ for various cases with $|a|=|b|=1$. 
Here the notation $\left(\begin{array}{c}n \\ m\end{array}\right)$ is shorthand for the binomial coefficient, namely the number of combinations of $n$ objects taken $m$ at a time. Do similar formulas exist for integer arguments greater than four? Numerous mathematicians have tried to find such formulas, but, until recently, none were known.

In 1997, using a combination of integer relation algorithms, the "Pade approximation" facility and some other computational techniques available in Mathematica and similar mathematical software systems, Jonathan Borwein (Peter Borwein's brother) and David Bradley discovered the following unanticipated general formula ([11] or [1, pg. 70-77]):

$$
\sum_{k=0}^{\infty} \zeta(4 k+3) x^{4 k}=\frac{5}{2} \sum_{k=1}^{\infty} \frac{(-1)^{k+1}}{k^{3}\left(\begin{array}{c}
2 k \\
k
\end{array}\right)\left(1-x^{4} / k^{4}\right)} \prod_{m=1}^{k-1}\left(\frac{1+4 x^{4} / m^{4}}{1-x^{4} / m^{4}}\right) .
$$

Here the notation $\prod_{m=1}^{k-1}$ means the product of the term to the right of $\prod$ for $m$ from 1 to $k-1$. Formula (13) permits one to read off an infinity of formulas for $\zeta(4 n+3)$, beginning with formula (11) above when $n=0$, simply by comparing coefficients of $x^{4 k}$ on the left-hand side and the right-hand side of (13).

In 2007, following a similar but much more deliberate computer-experimental procedure, as detailed in [5] or [1, pg. $70-77]$, a similar general formula was discovered for $\zeta(2 n+2)$ :

$$
\sum_{k=0}^{\infty} \zeta(2 k+2) x^{2 k}=3 \sum_{k=1}^{\infty} \frac{1}{k^{2}\left(\begin{array}{c}
2 k \\
k
\end{array}\right)\left(1-x^{2} / k^{2}\right)} \prod_{m=1}^{k-1}\left(\frac{1-4 x^{2} / m^{2}}{1-x^{2} / m^{2}}\right) .
$$

As with (13), one can now read off an infinity of formulas, beginning with formula (10) above when $n=0$. This general formula was then proved using what is known as the Wilf-Zeilberger algorithm [13]. A comparable general formula for $\zeta(2 n+4)$ has also been found, giving formula (12) above when $n=0$, but a similar general formula for all $\zeta(4 n+1)$ is not yet known.

It is worth emphasizing the fact that formula (14) above was both discovered and proven by computer. There is no reason for human mathematicians to panic, as considerable human ingenuity was involved in both steps. But this result is a harbinger of a future in which the computer is as essential to the mathematician, for both discovery and proof, as a particle collider is to a high-energy physicist, or as a DNA sequencer is to a molecular biologist.

\section{Proof versus experiment}

Although Wolfram repeatedly champions the experimental approach in $A$ New Kind of Science, he also acknowledges that experimental explorations are no substitute for rigorous proof. This principle has been amply underscored during the past few years by discoveries of some remarkable examples that serve as cautionary tales to those who too glibly apply experimental methods. 


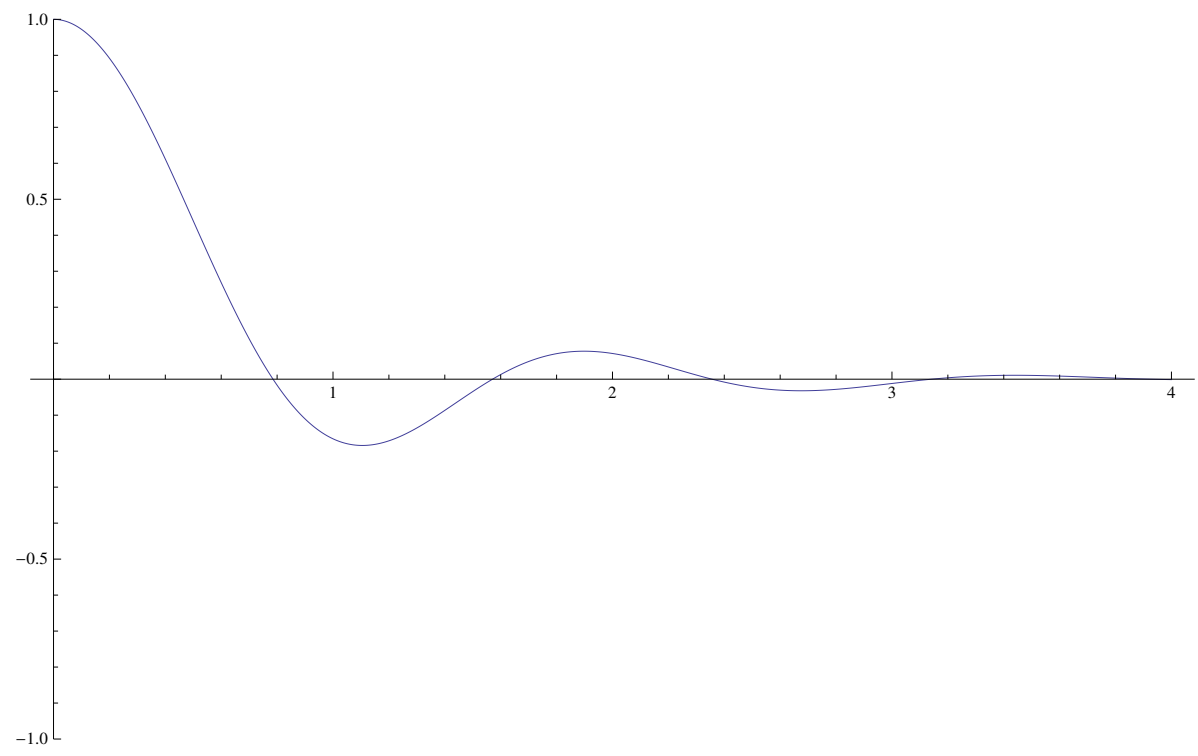

Figure 2: Graph of the oscillating function $\cos (2 x) \prod_{n=1}^{\infty} \cos (x / n)$

One particularly sobering example is the following:

$$
I=\int_{0}^{\infty} \cos (2 x) \prod_{n=1}^{\infty} \cos (x / n) \mathrm{d} x,
$$

where $\int_{0}^{\infty}$ means the usual signed area under the curve that we study in calculus. Calculating the numerical integral of this oscillating function (see Figure 2) to high accuracy is a nontrivial challenge, but can be done using a scheme described in [6]. When this integral was first computed to 20-digit accuracy, its value appeared to be $\pi / 8=0.392699 \ldots$. But when more than 50 digits were obtained, upon careful comparison with the numerical value of $\pi / 8$ :

$$
\begin{aligned}
I & =0.39269908169872415480783042290993786052464 \underline{5434187231595926} \ldots \\
\pi / 8 & =0.39269908169872415480783042290993786052464 \underline{6174921888227621} \ldots,
\end{aligned}
$$

it is clear that the two values disagree beginning with the 43rd digit! In other words, the integral $I$ is not $\pi / 8$. At first the authors of this study felt that there must be some "bugs" in the computer programs calculating the integral, but none were found.

Richard Crandall [12] later explained this mystery. In the course of analyzing physically motivated "running out of fuel" random walks, he showed that $\pi / 8$ is given by the following very rapidly convergent series expansion, of which the 
integral (15) above is merely the first term:

$$
\frac{\pi}{8}=\sum_{m=0}^{\infty} \int_{0}^{\infty} \cos [2(2 m+1) x] \prod_{n=1}^{\infty} \cos (x / n) \mathrm{d} x .
$$

As mentioned above, one term of this series is accurate to 43 digits; two terms are accurate to more than 500 digits; three series suffice for at least 8000 digits, and so on.

\section{Conclusion}

Considerable progress has been made since the publication of AKNS in identifying opportunities and techniques for experimental mathematics. New formulas have been discovered, interesting features have been identified in plots of mathematical structures, and computer-based techniques have been developed to prove certain types of results, as well as to discover them in the first place.

However, examples such the one mentioned in the previous section, where mathematical objects differ significantly from what one might think after performing an initial computation, draw attention to the fact that there has not yet been substantial and intellectually rigorous progress in the way experimental mathematics is presented in research papers, textbooks and classroom instruction, or in how the mathematical discovery process is organized. This is an arena where works such as A New Kind of Science can have significant impact. The present author, for one, looks forward to this dialogue. See [3] for more discussion. 


\section{References}

[1] D. Bailey, J. Borwein, N. Calkin, R. Girgensohn, R. Luke and V. Moll, Experimental Mathematics in Action, A K Peters, Natick, MA, 2007.

[2] David H. Bailey and Jonathan M. Borwein, "Experimental mathematics: Examples, methods and implications," Notices of the American Mathematical Society, vol. 52 (2005), pg. 502-514.

[3] David H. Bailey and Jonathan M. Borwein, "Exploratory experimentation and computation," Notices of the American Mathematical Society, vol. 58 (Nov 2011), pg. 1410-1419.

[4] David H. Bailey, Jonathan M. Borwein, Peter B. Borwein and Simon Plouffe, "The quest for Pi," Mathematical Intelligencer, vol. 19, no. 1 (Jan 1997), pg. $50-57$.

[5] David H. Bailey, Jonathan M. Borwein and David M. Bradley, "Experimental determination of Apery-like identities for $\zeta(2 n+2)$," Experimental Mathematics, vol. 15 (2006), pg. 281-289.

[6] D. H. Bailey, J. M. Borwein, V. Kapoor and E. Weisstein. "Ten Problems in Experimental Mathematics," American Mathematical Monthly, vol. 113, no. 6 (Jun 2006), pg. 481-409.

[7] David H. Bailey, Jonathan M. Borwein, Andrew Mattingly and Glenn Wightwick, "The computation of previously inaccessible digits of $\pi^{2}$ and Catalan's constant," Notices of the American Mathematical Society, to appear 11 Apr 2011, preprint available at http://crd-legacy.lbl.gov/ $\sim$ dhbailey/dhbpapers/bbp-bluegene.pdf.

[8] Bruce C. Berndt, Ramanujan's Lost Notebook, vol. 1-5, Springer, 1985 (date of vol. 1).

[9] Jonathan M. Borwein, "The Life of Pi," manuscript, 2011, available at http: //www. carma. newcastle.edu.au/ jb616/pi-2011.pdf.

[10] Jonathan M. Borwein and David H. Bailey, Mathematics by Experiment, AK Peters, Natick, MA, second edition, 2008.

[11] Jonathan M. Borwein and David M. Bradley, "Empirically determined Apery-like formulae for $\zeta(4 n+3)$," Experimental Mathematics, vol. 6 (1997), pg. 181-194.

[12] R. E. Crandall (2007). "Theory of ROOF Walks," 2007, available at http: //www . perfscipress. com/papers/ROOF11_psipress.pdf.

[13] H. S. Wilf and D. Zeilberger, "Rational functions certify combinatorial identities," Journal of the American Mathematical Society, vol. 3 (1990), pg. $147-158$. 
[14] Stephen Wolfram, A New Kind of Science, Wolfram Media, Champaign, IL 2002. 